\title{
PD/PID controller tuning based on model approximations: Model reduction of some unstable and higher order nonlinear models
}

\author{
Christer Dalen, David Di Ruscio \\ University College of Southeast Norway, P.O. Box 203, N-3901 Porsgrunn, Norway. E-mail: \\ David.Di.Ruscio@usn.no
}

\begin{abstract}
A model reduction technique based on optimization theory is presented, where a possible higher order system/model is approximated with an unstable DIPTD model by using only step response data. The DIPTD model is used to tune PD/PID controllers for the underlying possible higher order system. Numerous examples are used to illustrate the theory, i.e. both linear and nonlinear models. The Pareto Optimal controller is used as a reference controller.
\end{abstract}

Keywords: PD and PID controllers, tuning, double integrating system, time delay, maximum time delay error, relative time delay margin, robustness, performance, Pareto Optimal

\section{Introduction}

Many unstable plants and higher order systems may be controlled sufficiently well with PD/PID feedback controllers (p. 162, Silva et al. (2005)). One way of designing such control systems is to use a detailed firstprinciples model of the higher order and possible unstable system. The resulting models often contain a large number of states, integrators and also double integrators. This is at least the case in Mariner vessel models. Based on this higher order model there is for the moment no simple way to design a PID controller.

In this paper, it is proposed to approximate a Single Input Single Output (SISO) part of the higher order, possibly unstable and nonlinear Multiple Input Multiple Output (MIMO) system, with an Integrating Plus Time Delay (IPTD) model or Double IPTD (DIPTD) model. Initially, only PID controller tuning was considered, but for a shot at completion, we will also include PI controller tuning. We propose to use a simple step response of the underlying higher order SISO model or system in connection with optimization theory in order to develop the simplified (D)IPTD (IPTD or DIPTD) model between a control input, $u$, and output variable, $y=h_{p}(s) u$, where

$$
\begin{aligned}
& h_{p}(s)=K \frac{e^{-\tau s}}{s}, \\
& h_{p}(s)=K \frac{e^{-\tau s}}{s^{2}},
\end{aligned}
$$

where, $K$, is the gain- velocity or acceleration, i.o. and, $\tau$, is the time delay.

Hence, the approximating model parameters, $K$ and $\tau$, are simply obtained by minimizing the difference between the output of a step response of the higher order, possibly unstable and nonlinear model or system, and a step response of the simplified (D)IPTD model approximation in Eqs. (1) or (2), i.o. Notice, that the proposed method is a part of Prediction Error Methods (Ljung (1999)). Real observations of the underlying real system may also be used. Hence, instead of using a first-principles model, real process input-output data/observations of the plant are used.

By numerical investigations such approximations may be deduced from a short time interval, $t>\tau$, and 
that the (D)IPTD model approximation is sufficient to design a PI or PD/PID controller used to control and stabilize the system. The recently published $\delta$-tuning method (Di Ruscio and Dalen (2017)) is used in order to design the PD/PID controllers, and the earlier publication Di Ruscio (2010) for PI controllers.

The contributions in this paper may be itemized as follows:

- A model reduction technique to approximate higher order, possible unstable and nonlinear systems, with (D)IPTD models based on optimization over a possible short time interval is proposed and presented in Sec. 2.

- The simplified (D)IPTD model is used to design adequate PI or PD/PID controllers for sufficiently controlling and stabilizing the input-output behavior of the real plant.

- Numerous examples, both unstable and higher order systems, are used to illustrate the proposed design strategy. The examples are presented in Sec. 3.

All numerical calculations and plotting facilities are provided by using the MATLAB software, MATLAB (2016). The rest of this paper is organized as follows. In Sec. 2 we propose definitions and the model reduction technique for a (D)IPTD plant. Simulation examples are presented in Sec. 3. Lastly, discussion and concluding remarks are given in Sec. 4.

\section{Theory}

\subsection{Underlying Model}

Consider a continuous time SISO nonlinear state space model describing the dynamical system, viz.

$$
\begin{aligned}
\dot{x} & =f(x, u), \\
y & =g(x),
\end{aligned}
$$

where, $x \in \mathbb{R}^{n}$, is the state vector, $u \in \mathbb{R}$, is the control signal, $y \in \mathbb{R}$, is the output vector, and the vector functions, $x\left(t_{0}=0\right)$, is the initial state, $f(x, u) \in \mathbb{R}^{n}$, and, $g(x) \in \mathbb{R}$, are assumed Lipschitz continuous.

In this paper, noise is not considered, i.e. deterministic systems or models are assumed. A case with noise is suggested to be handled with a proper system identification method, e.g. Ljung (1999), Di Ruscio (1996) and Di Ruscio (2009), thereafter, possible (D)IPTD model approximations may be done for PI or PD/PID controller tuning.

\subsection{Definitions}

Consider the following PID controller on ideal/parallel form

$$
h_{c}(s)=K_{p}\left(1+\frac{1}{T_{i} s}+T_{d} s\right),
$$

where, $K_{p}, T_{i}$ and $T_{d}$ are the proportional constant, integral time and derivative time, i.o.

In order to compare the different controllers against each other we will consider indices such as defined in Åström and Hägglund (1995), Seborg et al. (1989) and Skogestad (2003).

For measuring performance in a feedback system as in Figure 1, the Integral Absolute Error $(I A E)$ is defined in the following, as

$$
I A E=\int_{0}^{\infty}|r-y| d t .
$$

Furthermore, from Eq. (6) we define:

- $I A E_{r}$ evaluates the performance in case of a step response in the reference, no disturbance.

- $I A E_{v u}$ evaluates the performance in case of a step input disturbance, with the reference equal to zero.

- $I A E_{v y}$ evaluates the performance in case of a step output disturbance, with the reference equal to zero.

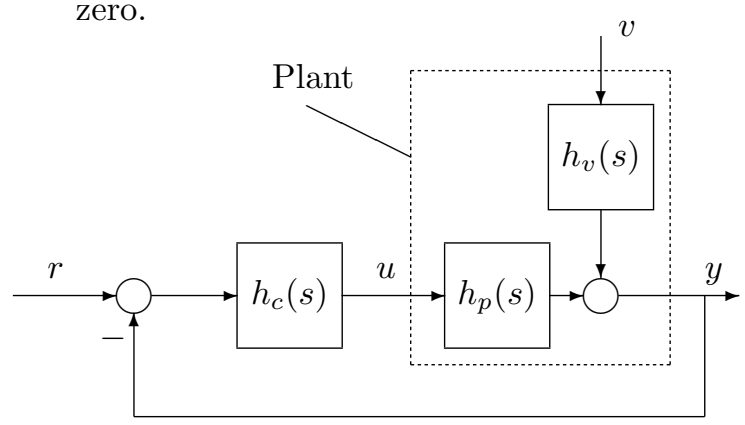

Figure 1: Control feedback system. Plant model, $h_{p}(s)$, disturbance model, $h_{v}(s)$, and controller, $h_{c}(s)$, in Eq. (5). Disturbance, $v$, at the input when, $h_{v}(s)=h_{p}(s)$, and at the output when, $h_{v}(s)=1$.

Robustness is quantified as in Garpinger and Hägglund (2014), i.e., $M_{s t}=\max \left\{M_{s}, M_{t}\right\}$, where

$$
M_{s}=\max _{\omega}\left|\frac{1}{1+h_{0}(j \omega)}\right|, M_{t}=\max _{\omega}\left|\frac{h_{0}(j \omega)}{1+h_{0}(j \omega)}\right|,
$$

where, $h_{0}(s)=h_{p}(s) h_{c}(s)$, is the loop transfer function.

Input usage is defined as in (Skogestad et al. (2002)),

$$
M_{k s}=\max _{\omega}\left|\frac{h_{p}(j \omega)}{1+h_{0}(j \omega)}\right| .
$$




\subsection{Model Reduction Techniques}

The half rule for model reduction, proposed by Skogestad (2003) can not be used to approximate unstable systems. It is proposed to use optimization theory to approximate a class/some higher order, possible unstable and nonlinear models as a (D)IPTD model, Eq. (1) or (2). Hence, an optimization method is used to find the two unknown parameters, i.e. the velocity/acceleration gain, $K$, and the time delay, $\tau$. However, it was observed in numerical investigations that introducing the final time, $t_{f}$, as a third unknown would give successful results.

Defining, $\rho=\left[K, \tau, t_{f}\right]$, in an optimal mean square sense we have that

$$
\hat{\rho}=\arg \min _{\rho} V(\rho) \text { s.t. }\left\{\begin{array}{l}
A \rho \leq b \\
l_{b} \leq \rho \leq u_{b}
\end{array},\right.
$$

where

$$
V(\rho)=\frac{1}{N\left(t_{f}\right)}\left(Y\left(t_{f}\right)-\widehat{Y}(\rho)\right)^{T} G\left(Y\left(t_{f}\right)-\widehat{Y}(\rho)\right),
$$

where, $Y\left(t_{f}\right) \in \mathbb{R}^{N}$, and, $\widehat{Y}(\rho) \in \mathbb{R}^{N}$, are the input step response time-series for the system/model and the (D)IPTD model, i.o., structured as vectors. $\mathrm{N}=$ length $\left(0: \mathrm{h}: t_{f}\right)$, i.e. in MATLAB notation, is the number of samples and, $h$, is the sampling interval.

Furthermore, in this paper, it is suggested to use the following

$$
\begin{aligned}
& A=\left[\begin{array}{ccc}
1 & 0 & 0 \\
0 & -1 & 0 \\
0 & 1 & -1
\end{array}\right], b=\left[\begin{array}{c}
c_{1} \\
-\frac{h}{2} \\
-2 h
\end{array}\right], \\
& l_{b}=\left[\begin{array}{c}
c_{1} \\
\frac{h}{2} \\
2 h
\end{array}\right], u_{b}=\left[\begin{array}{c}
c_{2} \\
\frac{L}{2} \\
\frac{L}{2}
\end{array}\right], G=I,
\end{aligned}
$$

where the upper bound on the time delay, $\tau$, and final time, $t_{f}$, corresponds to the pre-act time/derivative action (p. 190, Seborg et al. (1989)) setting, i.e. $\frac{L}{2}$ (p. 764, Ziegler and Nichols (1942)).

$L$ is, in this paper, defined as Ziegler's lag. See illustration in Figure 2. In some cases, where Ziegler's lag is not possible to identify, we will instead prescribe, $t_{f}$, i.e. we obtain a two-dimensional optimization problem. Note that, in Eq. (12), G, is the identity matrix. Notice, that the lower bound on the time delay $\tau$ is chosen according to Shannon's sampling theorem (Kotelnikov (1933), Shannon (1949)). The bounds for the gain $K$, i.e. $c_{1}<c_{2}$, need to be assigned from trial and error.

The optimization problem is solved by using fmincon (MATLAB (2016)) with default options, i.e. 'interior-point' algorithm.

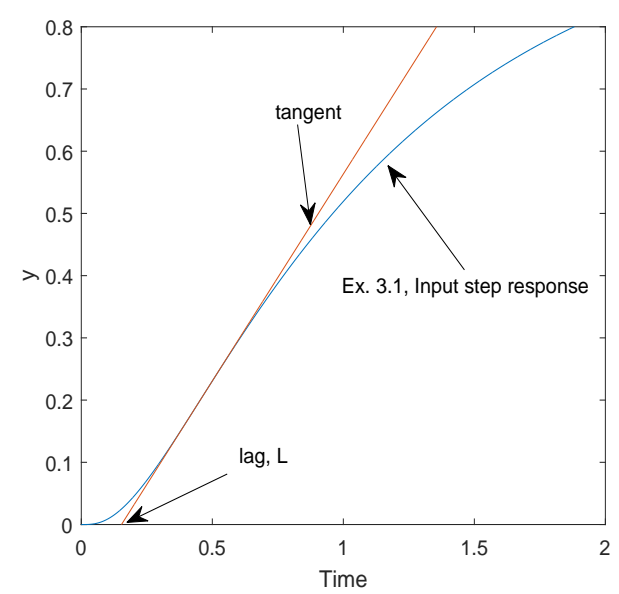

Figure 2: Illustration of the Ziegler's lag.

\section{Algorithm 2.1 (Simple $\delta$-PI or PD/PID tuning)}

1. Gather time series from open loop unit step response.

2. Obtain the (D)IPTD model approximation by solving the optimization problem given in Eq. (9).

3. Use the $\delta$-tuning method for PI or PD/PID controller tuning, i.e. Algorithm 6.1 in Di Ruscio (2010) or Algorithm 2.1 (include Eq. (27) for PID) p. 98 in Di Ruscio and Dalen (2017) for (D)IPTD i.o.

\subsection{The Pareto Optimal PID Controller}

For quantifying multiple performances, i.e. indices $I A E_{v u}$ and $I A E_{v y}$, consider the Pareto Optimal (PO) criterion (PO concept is presented in p. 60 Pareto $(1894 b))$

$$
J(p)=s_{r} \frac{I A E_{v y}(p)}{I A E_{v y}^{o}}+\left(1-s_{r}\right) \frac{I A E_{v u}(p)}{I A E_{v u}^{o}},
$$

where, $p=\left[K_{p}, T_{i}, T_{d}\right]$, and, $s_{r}=0.5$, is the servoregulator parameter, however it was suggested that, $0 \leq s_{r} \leq 1$ (p. 10, Di Ruscio (2012)).

The essential problem in generating the PO J vs. $M_{s t}$ vs. $M_{k s}$ trade-off surface, i.e. finding the $\mathrm{PO}$ controllers, renders in solving the following optimization problem given by

$$
\hat{p}=\arg \min _{p} J(p) \text { s.t. } c_{e q}(p)=0,
$$


where

$$
\begin{aligned}
c_{e q} & =\left[c_{s} ; c_{t}\right], \\
c_{s} & =M_{s t}-M_{s t}^{p r e}, \\
c_{t} & =M_{k s}-M_{k s}^{p r e},
\end{aligned}
$$

where, $M_{s t}^{p r e}$, is the prescribed robustness and, $M_{k s}^{p r e} \in$ $\mathbb{R}$, is the prescribed input usage. Notice, that this optimization problem is the same as considered in Jahanshahi et al. (2014), i.e. if we were to use Integral Squared Error instead of IAE.

Consider a two-dimensional optimization problem for stable processes, i.e. we generate a PO $J$ vs. $M_{s}$ trade-off curve, which is a similar problem as found in Balchen (1958) (i.e. if, $s_{r}=0$, in Eq. (13)). The index presented in Balchen (1958) can be seen common to IAE, however it is based on frequency response of the closed loop system. Furthermore, for stable processes, we will consider the range, $1.3 \leq M_{s} \leq 2.0$, i.e. the robust range suggested in Aström and Hägglund (1995). Note, that we will assume, $M_{s}>M_{t}$, for stable and, $M_{t}>M_{s}$, unstable processes, which is usually true (Skogestad and Postlethwaite (1996), Jahanshahi et al. (2014)).

It might be beneficial to measure how close to optimal, i.e. PO, a given tuning method is performing, hence the following mean square error criterion is introduced

$$
\Gamma_{m}(\theta)=\frac{1}{M}\left(J_{P O}-J_{m}(\theta)\right)^{T}\left(J_{P O}-J_{m}(\theta)\right),
$$

where, $J_{m}(\theta) \in \mathbb{R}^{M}$, is the performance vector generated from a given tuning method, $m$, with correspond$\operatorname{ing} \theta$ (i.e. generally free-parameter vector), $J_{P O} \in \mathbb{R}^{M}$, is the PO-PID performance vector previously solved, $\mathrm{M}=$ length $\left(J_{m}\right)$. E.g. for $\delta$-tuning, i.e. step 3. in Alg. 2.1, we may have that, $\theta=[K, \tau, c, \gamma]$, however, $\theta$, may be fixed as well. This is exercised in the coming sections and the numerical examples.

Lastly, we define an optimization problem

$$
\hat{\theta}=\arg \min _{\theta} \Gamma_{m}(\theta)
$$

where, $\Gamma_{m}$, is given in Eq. (18).

\subsection{Extending settings for $\delta$-tuning}

We propose some results for a possible extension of Sec 4.4 in Di Ruscio and Dalen (2017), i.e. choosing $\delta$-tuning PID parameters $c$ and $\gamma$.

Consider a DIPTD process model, where $K=1$ and $\tau=1$, i.e. $\theta=[c, \gamma]$. The reference controllers, i.e. the optimal output PD controller and the optimal input disturbance PID controller are given in Table 1. The solution to the optimization problem in Eq. (19) is given in row 1 in Table 4 . Interestingly, we obtain, $\hat{\theta}=[2.12,2.12]$, i.e. cf. Di Ruscio and Dalen (2017) where, $\hat{\theta}^{*}=[2.24,2.24]$ ( $^{*}$ means the $J_{P O}$ in Eq. (18) is substituted by $\delta$-optimal $J$ curve, i.e. the optimization problem is solved per $M_{s}$ instead of the full range). Hence, considering the reference example, this change in settings can be thought of as rotating the $\delta$-tuning trade-off curve counter-clockwise around the point $(1.54,1.56)$, see Figure 3 .

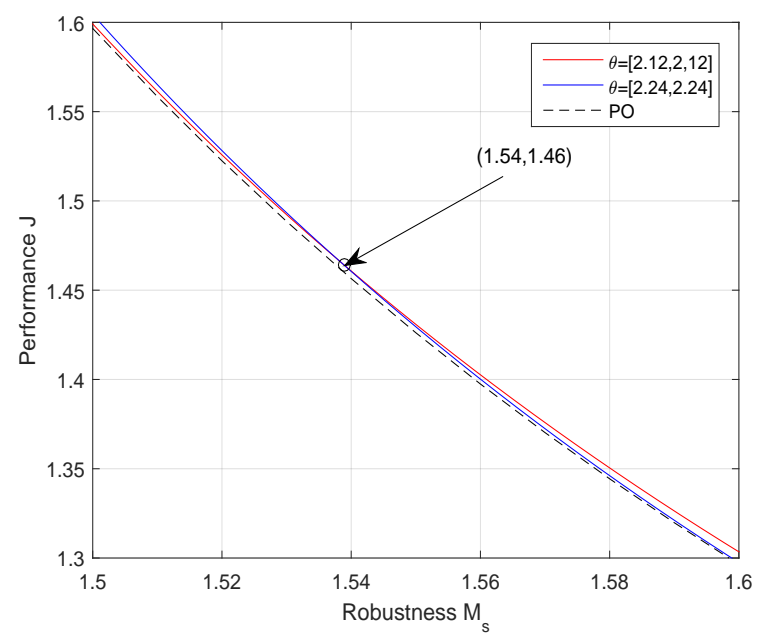

Figure 3: Reference Example: Performance vs. robustness, J vs. $M_{s}$ trade-off curves. Comparing settings, $\hat{\theta}=[2.12,2.12]$, and $\hat{\theta}^{*}=$ $[2.24,2.24]$ vs. the PO curve.

Table 1: Shows the reference controllers which minimize the indices, $I A E_{v y}$, and, $I A E_{v u}$, i.e. optimal output and input disturbance (ideal) $\mathrm{PD} / \mathrm{PID}$ controllers as in Eq. (5), i.o., given a prescribed, $M_{s}=1.59$. DIPTD process where, $K=1, \tau=1$. The weights, $I A E_{v y}^{o}=$ $I A E_{v u}^{o}=1$, in Eq. (13).

\begin{tabular}{|l|l|l|}
\hline$s_{r}$ & $\hat{p}$ & $J_{A l g .2 .1}(\hat{p})$ \\
\hline \hline 1 & $0.02, \infty, 25.84$ & 4.15 \\
\hline 0 & $0.07,9.15,5.41$ & 169.09 \\
\hline
\end{tabular}

\section{Numerical Examples}

We seek to justify the proposed method, Algorithm (Alg.) 2.1, in the coming sections by using a good variety of models with stable and unstable nature. We will first demonstrate the proposed method on linear process examples, thereafter we consider unstable nonlinear models. 
Table 2: Settings for the $\delta$-tuning PID parameters $c$ and $\gamma$ in Alg. 2.1 and Eq. (27) in Di Ruscio and Dalen (2017). row 1) optimized over the robust region and 2) over the entire region. The solution of the optimization problem in Eq. (19).

\begin{tabular}{|l|l|l|l|}
\hline Option & $\hat{\theta}$ & $\Gamma_{\text {Alg.2.1 }}(\hat{\theta})$ & Region \\
\hline \hline 1 & $2.12,2.12$ & $1.8323 \mathrm{E}-4$ & $1.3 \leq M_{s} \leq 2.0$ \\
\hline 2 & $2.24,2.24$ & $7.8248 \mathrm{E}-4$ & $1.3 \leq M_{s} \leq 2.0$ \\
\hline
\end{tabular}

\subsection{Linear Examples}

We start by studying some of the linear process model examples given in Seborg et al. (2004) or Skogestad (2003). We will compare Alg. 2.1 vs. some existing model-based PID tuning methods, i.e. the Simple Internal Model Control (SIMC) (Skogestad (2003)) and the Korean-SIMC (K-SIMC) method (Lee et al. (2014)).

\section{Example 3.1 (Fourth-Order Model)}

Consider the fourth-order process model studied in exercise 12.7 on p. 331 in Seborg et al. (2004) and Example E5 in Skogestad (2003), i.e. the transfer function in row 1 and column (col.) 2 in Table 3.

The Second Order Plus Time Delay (SOPTD) model approximations (sampling interval $h=0.001$ ) for the SIMC and K-SIMC methods are given in rows 1:2, and col. 3 in Table 6, i.o. The DIPTD model approximation, i.e. step 2 in Alg. 2.1, is given in row 1 and col. 2 in Table 5, where $c_{1}=1$ and $c_{2}=10$ are chosen as bounds for the gain.

In this example, Alg. 2.1 is shown superior to the SIMC and K-SIMC method, viz. rows 1:3 and col. 4 in Table 6 shows that that Alg. 2.1 is $\frac{\Gamma_{S I M C}}{\Gamma_{\text {Alg.2.1 }}}=4.0$ times better than the runner up, SIMC. The corresponding $J$ vs. $M_{s}$ trade-off curves are shown in Figure 4.

\section{Example 3.2 (Bioseperation Process)}

A multistage bioseperation process, as motivated in exercise 7.4 on p. 183 in Seborg et al. (2004), may be described by the transfer function in Table 3 (row 2, col. 2).

The SOPTD model approximations $(h=0.01)$ for the SIMC and K-SIMC methods are given in Table 6 (rows 4:5, col. 3). The DIPTD model approximation $\left(c_{1}=0.01, c_{2}=1\right.$ ) is given in Table 5 (row 2 and col. 2).

Alg. 2.1 is observed to be superior compared to the other model based methods, i.e. in Table 6 (rows 4:6, col. 4) it is seen that Alg. 2.1 is $\frac{\Gamma_{S I M C}}{\Gamma_{\text {Alg.2.1 }}}=8.5$ times better than the runner up, SIMC. The J vs. $M_{s}$ tradeoff curves are shown in Figure 5.

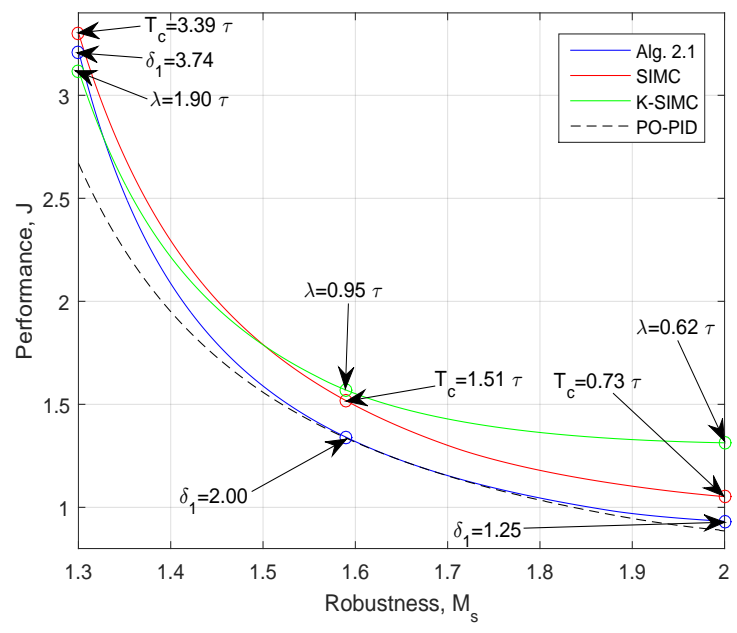

Figure 4: Example 3.1: Performance vs. robustness, $\mathrm{J}$ vs. $M_{s}$ trade-off curves. Comparing Alg. 2.1, SIMC and K-SIMC vs. the PO-PID controllers for the process model at row 1 , col. 2 in Table 3. The weights for the PO criterion in Eq. (13) are given in Table 5. The performance measures, i.e. $\Gamma_{m}$, Eq. (18), are given in Table 6.

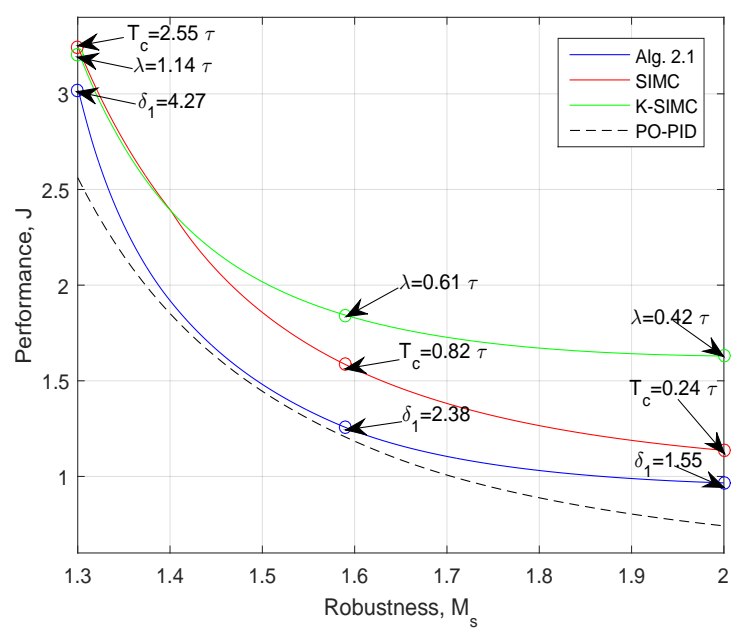

Figure 5: Example 3.2: Performance vs. robustness, $\mathrm{J}$ vs. $M_{s}$ trade-off curves. Comparing Alg. 2.1, SIMC and K-SIMC vs. the PO-PID controllers for the process model at row 2, col. 2 in Table 3. The servo-regulator, $s_{r}=0.5$, and the weights for the PO criterion in Eq. (13) are given in Table 5. The method performance measures, i.e. $\Gamma_{m}$ in Eq. (18), are given in Table 6. 


\section{Example 3.3 (Positive Numerator)}

Consider the process model with a positive numerator as studied in Example 4 in Aström et al. (1998) and Example E3 Skogestad (2003), i.e. the transfer function given in Table 3 (row 3, col. 2).

The SOPTD model approximations $(h=0.01)$ for the SIMC and K-SIMC method are shown in Table 6 (rows 7:8, col. 3) The DIPTD model approximation $\left(c_{1}=1, c_{2}=10\right)$ is given in Table 5 (row 3, col. 2).

Notice, that the gain, $K$, in the K-SIMC method is a function of the tuning parameter, i.e., $K=f(\lambda)$, where, $\lambda$, is similar to the tuning parameter, $T_{c}$, in SIMC.

Alg. 2.1 gives the best performance, viz. from $\mathrm{Ta}$ ble 6 (rows 7:9, col. 4) we have that Alg. 2.1 is $\frac{\Gamma_{S I M C}}{\Gamma_{\text {Alg.2.1 }}}=2.9$ times better than the runner up, SIMC. The corresponding $J$ vs. $M_{s}$ trade-off curves are shown in Figure 6.

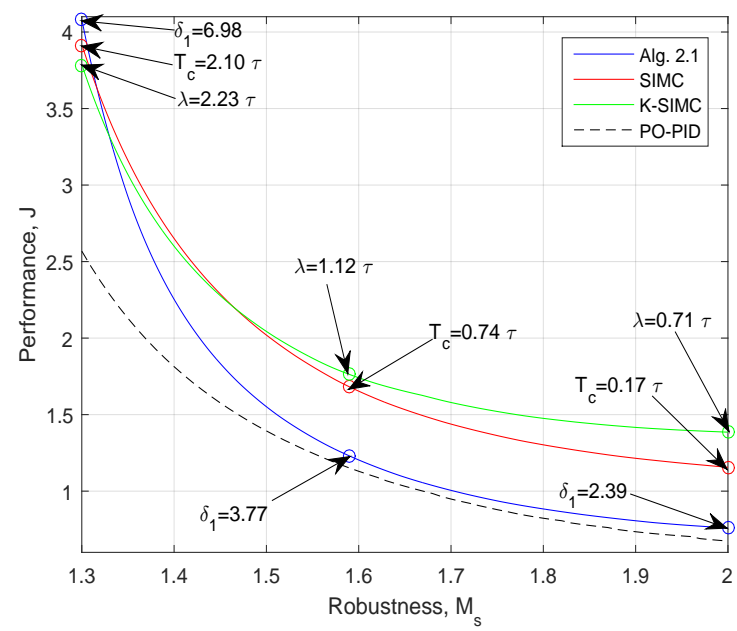

Figure 6: Example 3.3: Performance vs. robustness, $\mathrm{J}$ vs. $M_{s}$ trade-off curves. Comparing Alg. 2.1, SIMC and K-SIMC vs. the PO-PID controllers for the process model at row 3, col. 2 in Table 3. The servo-regulator, $s_{r}=0.5$, and the weights for the PO criterion in Eq. (13) are given in Table 5. The method performance measures, i.e. $\Gamma_{m}$ in Eq. (18), are given in Table 6.

\section{Example 3.4 (Quadruple Poles)}

Consider the transfer function with quadruple poles in Table 3 (row 4, col. 2).

The SOPTD model approximations $(h=0.01)$ for SIMC and K-SIMC methods are given in Table 6 (rows 10:11, col. 3). The DIPTD model approximation $\left(c_{1}=\right.$ $1, c_{2}=10$ ) is given in Table 5 (row 4, col. 2).

Alg. 2.1 is shown superior, i.e. from Table 6 (rows 10:12, col. 4) we have that Alg. 2.1 is $\frac{\Gamma_{K-S I M C}}{\Gamma_{A l g .2 .1}}=13.0$ times better than the runner up, K-SIMC. The $J$ vs. $M_{s}$ trade-off curves are shown in Figure $\%$

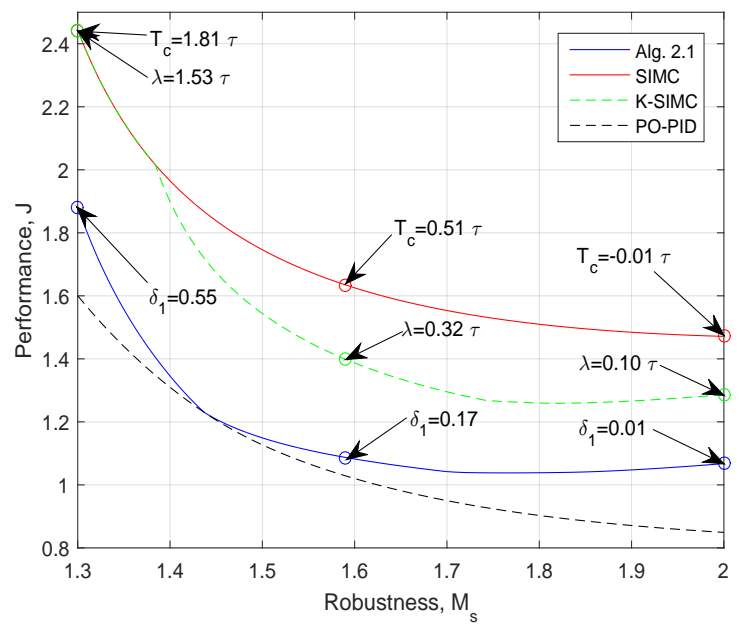

Figure 7: Example 3.4: Performance vs. robustness, $\mathrm{J}$ vs. $M_{s}$ trade-off curves. Comparing Alg. 2.1, SIMC and K-SIMC vs. the PO-PID controllers for the process model at row 4 , col. 2 in Table 3. The servo-regulator, $s_{r}=0.5$, and the weights for the PO criterion in Eq. (13) are given in Table 5. The method performance measures, i.e. $\Gamma_{m}$ in Eq. (18), are given in Table 6 .

\section{Example 3.5 (Perfectly Stirred Tank)}

This example is taken from Exercise 14.3. on p. 334 in Seborg et al. (1989). A perfectly stirred tank heating a flowing liquid is described by the process model in Table 3 (row 5, col. 2), i.e. a transfer function from power applied to the heater to the measured temperature.

The SOPTD model approximations $(h=0.01)$ for SIMC and K-SIMC methods are given in Table 6 (rows 13:14, col. 3), i.o. The DIPTD model approximation is shown in ( $c_{1}=1, c_{2}=10$ ) Table 5 (row 5, col. 2).

Alg. 2.1 has an edge over the other model based techniques, i.e. in Table 6 (rows 13:15, col. 4) observe that Alg. 2.1 is $\frac{\Gamma_{S I M C}}{\Gamma_{\text {Alg. } 2.1}}=2.4$ times better than the next best, SIMC. The corresponding J vs. $M_{s}$ trade-off curves are shown in Figure 8.

\section{Example 3.6 (Pipeline-Riser System)}

The severe-slugging flow regime is a common problem at the oil fields and it is characterized by large oscillations in pressure and flow rates. Active control of the topside choke is the recommended solution (Yocum (1973) and Schmidt Z. (1979)).

A schematic model describing a pipeline-riser system is given in Figure 9. The inflow rates of liquid and gas to the system, $w_{l}$ and $w_{g}$, i.o., are assumed to be independent disturbances. Based on experimental data, 


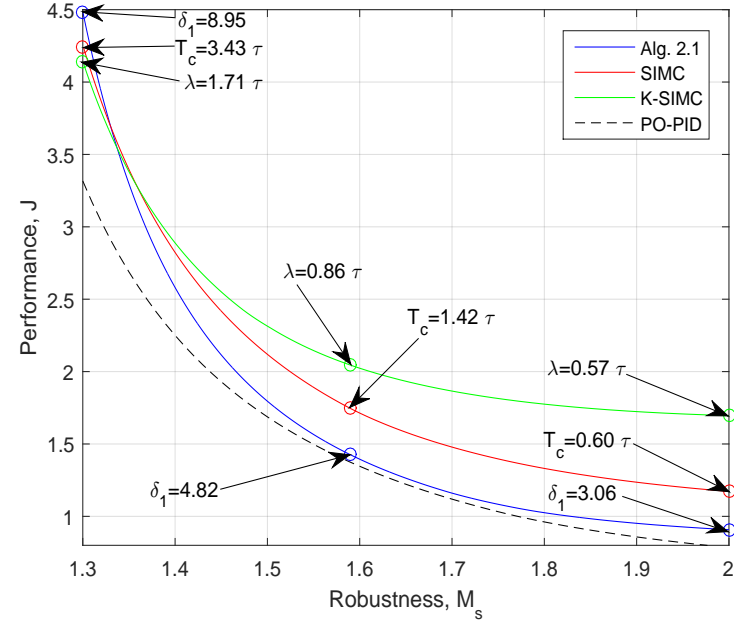

Figure 8: Example 3.5: Performance vs. robustness, $\mathrm{J}$ vs. $M_{s}$ trade-off curves. Comparing Alg. 2.1, SIMC and K-SIMC vs. the PO-PID controllers for the process model at row 5, col. 2 in Table 3. The servo-regulator, $s_{r}=0.5$, and the weights for the PO criterion in Eq. (13) are given in Table 5 . The method performance measures, i.e. $\Gamma_{m}$ in Eq. (18), are given in Table 6.

it was shown in Jahanshahi and Skogestad (2013) that such a pipeline-riser system can be described by the process model in Table 3 (row 6, col. 2), i.e. a transfer function from the topside choke, $u$, to inlet pressure, $y$.

The DIPTD model approximation, i.e. step 2 in Alg. 2.1, where $c_{1}=-10$ and $c_{2}=-0.001$, are given in Table 5 (row 6, col. 2). The IMC PID plus derivative Filter (IMC-PIDF) method in Jahanshahi et al. (2014) is based on the exact model, in Figure 9.

Note, that for this application it is crucial for the dynamics to have a low-pass filter on the derivative action (Jahanshahi et al. (2014)), hence we have the following PIDF controller

$$
h_{c}(s)=K_{p}\left(1+\frac{1}{T_{i} s}+\frac{T_{d} s}{T_{f} s+1}\right),
$$

where $T_{f}=4$, cf. the numerator in the process model.

This filter is also added to Alg. 2.1, hence Alg. 2.1 (PIDF) is written in instead.

The trade-off PO surface together with the curves for Alg. 2.1 (PIDF) and IMC-PIDF are shown in Figure 10. See also Figure 11 for equivalent sets of $2 D$ plots.

Note, that for making Alg. 2.1 (PIDF) competitive we have used, $c=\gamma=4.24$, i.e. 2 times our suggested settings for this case.

Alg. 2.1 (PIDF) was found superior in terms of $\Gamma_{m}$, viz. Alg. 2.1 (PIDF) is $\frac{\Gamma_{I M C-P I D F}}{\Gamma_{A l g .2 .1(P I D F)}}=18.1$ times better than IMC-PIDF, however it might be argued that a better criterion may exist.

Converting the Alg. 2.1 (PIDF) controller in row 6, col. 2 in Table 8, where $\tau=6.66$, to $K_{c}=$ $K_{p}=-11.87, K_{i}=\frac{K_{p}}{T_{i}}=-1.38$ and $K_{d}=K_{p} T_{d}=$ -152.77 , we see that these are approximately equal to the controller proposed in Table 1 row 1 in Jahanshahi et al. (2014).

Note, that we propose to use PO-PIDF controller for prescribed robustness $M_{t}=1.4$ instead of $M_{t}=1.15$, as in Jahanshahi et al. (2014). See row 6, col. 3 in Table 8 .

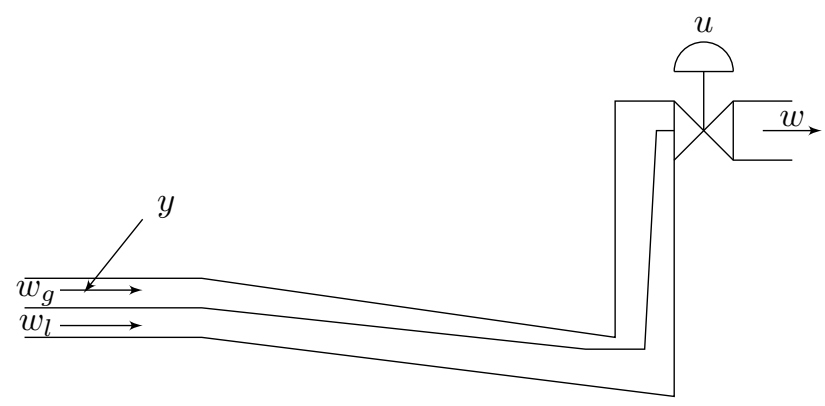

Figure 9: Example 3.6. Schematic model of the pipeline-riser, viz. the plant in the feedback system in Figure 1.

Table 3: Examples and the corresponding linear process models for Sec. 3.1.

\begin{tabular}{|l|l|}
\hline Example & Process model, $h_{p}(s)$ \\
\hline \hline 3.1 & $\frac{1}{(s+1)(0.2 s+1)(0.04 s+1)(0.008 s+1)}$ \\
\hline 3.2 & $\frac{2}{(5 s+1)(3 s+1)(s+1)}$ \\
\hline 3.3 & $\frac{2(15 s+1)}{(20 s+1)(s+1)(0.1 s+1)^{2}}$ \\
\hline 3.4 & $\frac{1}{(s+1)^{4}}$ \\
\hline 3.5 & $\frac{10}{(s+1)(5 s+1)(0.2 s+1)}$ \\
\hline 3.6 & $\frac{-0.0098(s+0.25)}{s^{2}-0.04 s+0.025}$ \\
\hline
\end{tabular}

\subsection{Nonlinear Examples}

This section contains results on PID controller implementation on a good variety of nonlinear models with unstable nature. Note, that we are only given open loop data, hence model reduction techniques as the half rule method (Skogestad (2003)) can not be used, however we will instead use Alg. 2.1 where step 3. is 


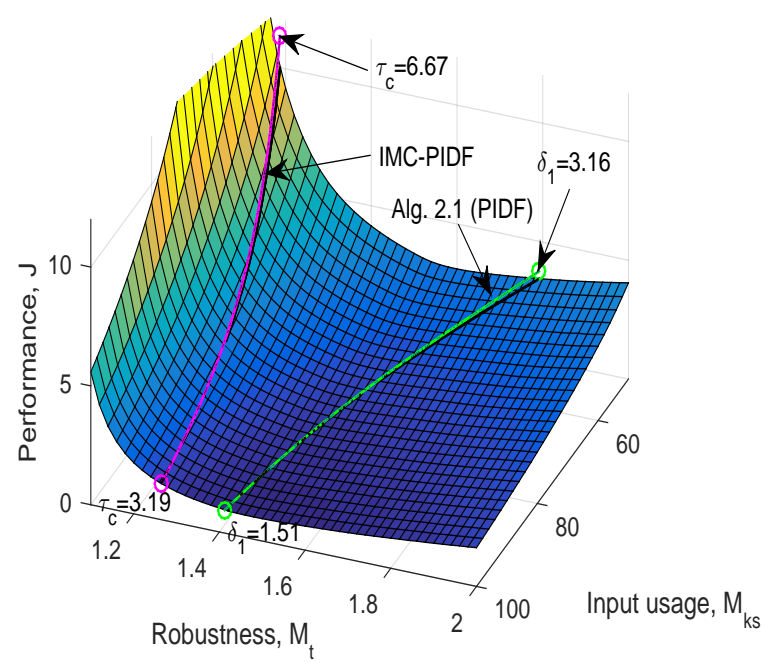
vs. the PO-PID controllers for the process model at row 6 , col. 2 in Table 3. The servo-regulator, $s_{r}=0.5$, and the weights for the PO criterion in Eq. (13) are given in Table 5.

Table 4: Examples and the corresponding reference controllers, i.e. the optimal input and output performances for prescribed $M_{s t}=1.59$ or input usage $M_{k s}=100$, (i.e. only 'and' for col. 6).

\begin{tabular}{|l|l|l|}
\hline Example & $I A E_{v y}^{o}$ & $I A E_{v u}^{o}$ \\
\hline \hline 3.1 & 0.0847 & 0.0133 \\
\hline 3.2 & 1.2877 & 0.7215 \\
\hline 3.3 & 0.1074 & 0.0250 \\
\hline 3.4 & 1.9054 & 1.5851 \\
\hline 3.5 & 0.3020 & 0.2740 \\
\hline 3.6 & 2.0855 & 0.0380 \\
\hline
\end{tabular}

Table 5: Results from Sec. 3.1. Step 2 in Alg. 2.1, i.e. optimal DIPTD model approximation.

\begin{tabular}{|l|l|l|}
\hline Example & $\hat{\rho}$ & $V(\hat{\rho})$ \\
\hline \hline 3.1 & $3.9559,0.0249,0.0578$ & $1.3515 \mathrm{E}-8$ \\
\hline 3.2 & $0.0907,0.3061,0.6928$ & $2.4642 \mathrm{E}-7$ \\
\hline 3.3 & $4.8212,0.0193,0.0530$ & $4.0529 \mathrm{E}-8$ \\
\hline 3.4 & $0.9164,0.4900,0.6079$ & $6.4056 \mathrm{E}-7$ \\
\hline 3.5 & $1.0205,0.0602,0.3295$ & $1.0444 \mathrm{E}-7$ \\
\hline 3.6 & $-0.0934,0.1181,0.5243$ & $1.4997 \mathrm{E}-6$ \\
\hline
\end{tabular}

Figure 11: Example 3.6: $J$ vs. $M_{k s}$ and $J$ vs. $M_{t}$ trade-off curve plots. Comparing Alg. 2.1, IMC-PIDF vs. the PO-PID controllers for the process model at row 6 , col. 2 in Table 3. col. 1 is Alg. 2.1 (PIDF) and col. 2 is IMC-PIDF.
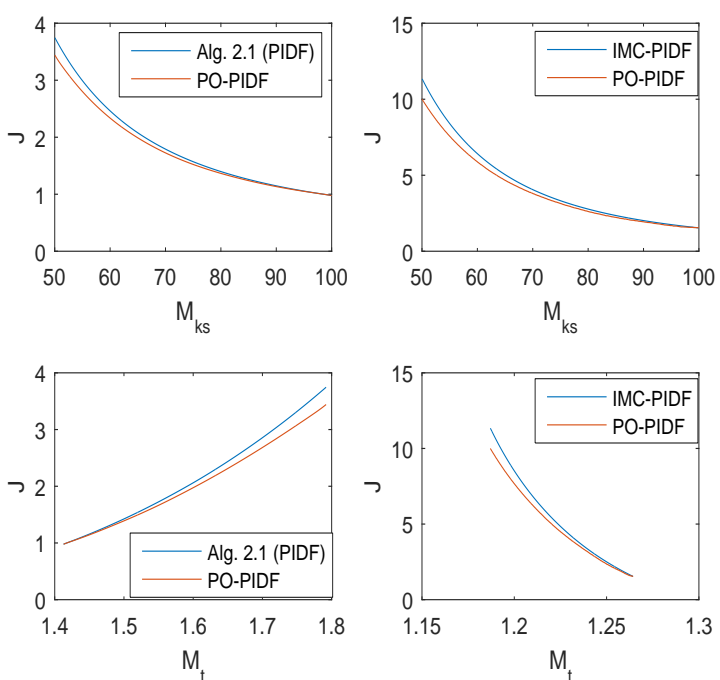

Table 6: Summarised results from Sec. 3.1. Comparing SIMC, K-SIMC and the proposed Alg. 2.1, based on model reduction to SOPTD $\left(K, \tau, T_{1}, T_{2}\right)$ or DIPTD $(K, \tau)$ models. † means that the model approximation is given in col. 2 in Table 5 for the corresponding example.

\begin{tabular}{|c|l|l|l|}
\hline Ex & Method & Approximation & $\Gamma_{m}$ \\
\hline \hline 3.1 & SIMC & $1,0.0285,1,0.2200$ & 0.0628 \\
\hline & K-SIMC & $1,0.0446,1,0.2040$ & 0.0966 \\
\hline & Alg. 2.1 & $\dagger$ & 0.0156 \\
\hline 3.2 & SIMC & $2,0.5050,5,3.5$ & 0.1899 \\
\hline & K-SIMC & $2,0.8471,5,3.1667$ & 0.4947 \\
\hline & Alg. 2.1 & $\dagger$ & 0.0224 \\
\hline 3.3 & SIMC & $1.5,0.05,1,0.15$ & 0.4255 \\
\hline & K-SIMC & $f(\lambda), 0.05,1,0.15$ & 0.5142 \\
\hline & Alg. 2.1 & $\dagger$ & 0.1489 \\
\hline 3.4 & SIMC & $1,1.5050,1,1.5$ & 0.4000 \\
\hline & K-SIMC & $1,1.6717,1,1.5$ & 0.2068 \\
\hline & Alg. 2.1 & $\dagger$ & 0.0159 \\
\hline 3.5 & SIMC & $10,0.1050,5,1.1000$ & 0.2165 \\
\hline & K-SIMC & $10,0.1854,5,1.0200$ & 0.5637 \\
\hline & Alg. 2.1 & $\dagger$ & 0.0885 \\
\hline 3.6 & Alg. 2.1 (PIDF) & $\dagger$ & 0.0187 \\
\hline & IMC-PIDF & Exact model & 0.3392 \\
\hline
\end{tabular}


Table 7: Results from Sec. 3.1. Margins organized as $(G M, P M, D M)$ for Alg. 2.1 and runner-up, i.e. SIMC, with exception of row 4 , K-SIMC, and the optimal controller, PO-PID, for prescribed robustness, $M_{s t}=1.59$ or input usage $M_{k s}=50$, (i.e. only 'and' for col. 6).

\begin{tabular}{|r|r|r|r|}
\hline Ex & \multicolumn{1}{|l|}{ Alg. 2.1 } & \multicolumn{1}{|c|}{ Runner-up } & \multicolumn{1}{l|}{ PO-PID } \\
\hline \hline 3.1 & $8.05,48.55,0.06$ & $8.81,46.67,0.06$ & $8.18,47.94,0.06$ \\
\hline 3.2 & $\infty, 45.82,0.79$ & $\infty, 45.49,0.85$ & $\infty, 46.69,0.71$ \\
\hline 3.3 & $\infty, 47.26,0.05$, & $\infty, 44.81,0.07$ & $\infty, 47.11,0.05$ \\
\hline 3.4 & $4.47,56.22,1.68$ & $4.76,58.88,2.20$ & $4.32,65.44,1.91$ \\
\hline 3.5 & $\infty, 45.15,0.18$ & $\infty, 43.49,0.21$ & $\infty, 45.28,0.18$ \\
\hline 3.6 & $0.25,45.97,1.67$ & $0.11,75.74,2.49$ & $0.17,64.30,2.11$ \\
\hline
\end{tabular}

Table 8: Results from Sec. 3.1. PID controllers organized as $\left(K_{p}, T_{i}, T_{d}\right)$ for Alg. 2.1 and runnerup, i.e. SIMC, with exception of row $4, \mathrm{~K}$ SIMC, and the optimal controller, PO-PID, for prescribed robustness, $M_{s t}=1.59$ or input usage $M_{k s}=50$, (i.e. only 'and' for col. $6)$.

\begin{tabular}{|l|l|c|c|}
\hline Ex & Alg. 2.1 & Runner-up & PO-PID \\
\hline \hline 3.1 & $24.3,0.32,0.15$ & $24.7,0.51,0.12$ & $24.5,0.30,0.14$ \\
\hline 3.2 & $5.54,4.36,2.05$ & $5.31,7.17,1.79$ & $5.56,3.40,2.54$ \\
\hline 3.3 & $13.2,0.39,0.18$ & $11.0,0.50,0.10$ & $13.3,0.24,0.17$ \\
\hline 3.4 & $1.79,2.41,1.14$ & $1.38,3.06,0.67$ & $1.82,2.62,1.33$ \\
\hline 3.5 & $4.29,1.48,0.70$ & $4.09,2.12,0.53$ & $4.27,1.00,0.73$ \\
\hline 3.6 & $-30.4,5.49,1.30$ & $-11.9,8.59,12.9$ & $-21.2,5.09,5.45$ \\
\hline
\end{tabular}

substituted with SIMC PID tuning, i.e. denoted Alg. 2.1 (SIMC).

We define Total Value (TV) index formulated in discrete time as

$$
T V=\sum_{k=1}^{\infty}\left|\Delta u_{k}\right|
$$

where, $\Delta u_{k}=u_{k}-u_{k-1}$, is the control rate of change, and, $k$, is discrete time.

\section{Example 3.7 (Inverted pendulum-cart system)}

In this example, we will consider an inverted pendulum system, which is one of the most used systems for benchmarking various control strategies. The reason being that the structure renders as both relatively simple and rich (Boubaker (2012)).

It may be shown that the dynamics of an inverted pendulum on a cart with mass can be described using the model in Eqs. (3)-(4), where the RHS is substituted by the following,

$$
\begin{aligned}
f_{1} & =x_{2} \\
f_{2} & =b\left(g\left(1+m_{1}+m_{2}\right) \sin \left(x_{1}\right)-u \cos \left(x_{1}\right)\right) \\
g & =x_{1}
\end{aligned}
$$

where,

$$
b=\frac{\left(m_{1}+m_{2}\right) l_{g}}{J_{t}+\left(m_{1}+m_{2}\right) l_{g}^{2}} .
$$

In Eq. (25), the moment of inertia of the rod and the distance to center of mass are expressed as

$$
\begin{aligned}
J_{t} & =m_{2} \frac{l^{2}-3 l l_{g}+3 l_{g}^{2}}{3}+m_{1}\left(l-l_{g}\right)^{2} \\
l_{g} & =\frac{l}{2} \frac{m_{1}+2 m_{2}}{m_{1}+m_{2}}
\end{aligned}
$$

i.o., where $l=0.7$ is the length, $m_{2}=0.044$, is the mass of the pendulum arm (neglecting the load), $m_{1}=0.2$, is the mass of the load and $g=9.81$ is the gravitational constant. A schematic model of the inverted pendulum on a cart is shown in Figure 12.

Furthermore, we define the input-output case as,

$u \in \mathbb{R}:=\{u:$ force exerted on the cart $(N)$,

$y \in \mathbb{R}:=\{y:$ tilt angle (rad).

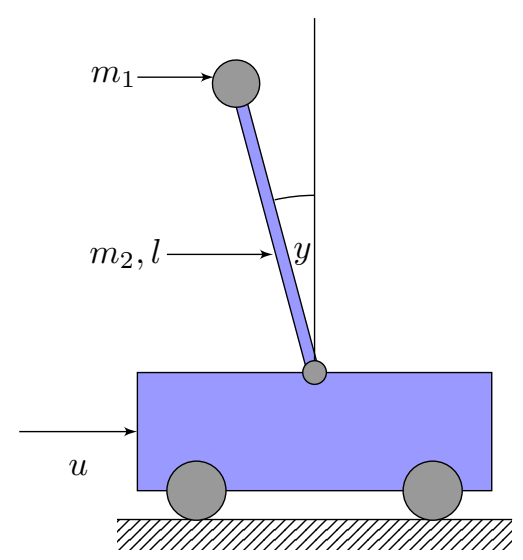

Figure 12: Example 3.7. Schematic model of the inverted pendulum on a cart, i.e. the plant in the feedback system in Figure 1. Note, that arrows may have different meanings, i.e. labels and vector force $u$.

The optimal DIPTD model approximation is given in Table 9 (col. 2).

Table 9: Example 3.7. Results from step 2 in Alg. 2.1, i.e. optimal DIPTD model approximation, where $c_{1}=0$ and $c_{2}=10$.

\begin{tabular}{|l|l|l|}
\hline \hline Method & $\hat{p}$ & $V(\hat{p})$ \\
\hline \hline Alg. 2.1 & $-1.8252,0.0139,0.2423$ & $2.3618 \mathrm{E}-7$ \\
\hline
\end{tabular}

Zero tilt angle is desired, at Time $=0.1 \mathrm{~s}$, we give the pendulum an output step $v_{v y}=\frac{\pi}{30} \mathrm{rad}(6 \mathrm{deg})$, and at, 
Time $=2.0 \mathrm{~s}$, we introduce an input step disturbance where $v_{v u}=20 \mathrm{~N}$. The time-series are shown in Figure 13.

From Table 10, we see that Alg. 2.1 outperforms Alg. 2.1 (SIMC) in terms of (sum) IAE. Furthermore, we see that Alg. 2.1 is $\frac{I A E_{v u}^{A l g .2 .1(S I M C)}}{I A E_{v u}^{A l g .2 .1}}=1.4$ times better in terms of $I A E_{v u}$, however Alg. 2.1 (SIMC) is found $\frac{I A E_{v u}^{A l g .2 .1}}{I A E_{v y}^{A l g .2 .1(S I M C)}}=1.2$ times better in terms of IAE $E_{v y}$.
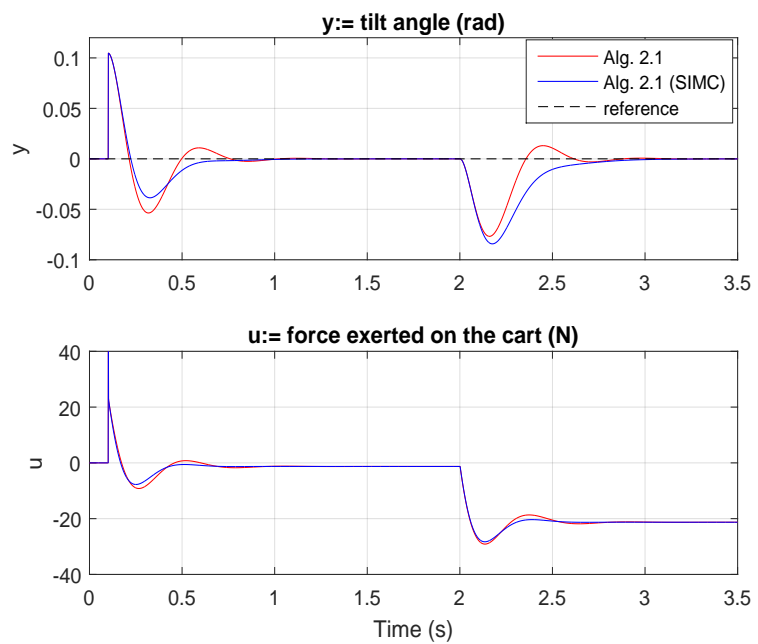

Figure 13: Example 3.7. Simulation of the inverted pendulum nonlinear model with PID control. Output and input step disturbance at, Time $=0.1 \mathrm{~s}$ and, Time $=2.0 \mathrm{~s}$, i.o. Input $u$ is limited.

Table 10: Example 3.7. Shows the indices $I A E_{v y}$, $I A E_{v u}, I A E$ (sum) and $T V$ for Alg. 2.1 where $\delta=1.6$, and Alg. 2.1 (SIMC) where $T_{c}=1.5 \tau$.

\begin{tabular}{|l|l|l|l|l|}
\hline \hline Method & $I A E_{v y}$ & $I A E_{v u}$ & $I A E$ & $T V$ \\
\hline \hline Alg. 2.1 & 0.0182 & 0.0178 & 0.0360 & 184.7389 \\
\hline Alg. 2.1 (SIMC) & 0.0158 & 0.0245 & 0.0403 & 171.6880 \\
\hline
\end{tabular}

\section{Example 3.8 (Segway)}

A commercial example of a two-wheel system is the Segway, which has been experiencing a growth of popularity as an eco-friendly alternative for short journeys. The dynamics of a Segway are presented in LIngenieur (2005), which we have put on an implicit state space form, viz.

$$
\begin{aligned}
M(x) \dot{x} & =f(x, u) \\
y & =g(x),
\end{aligned}
$$

$$
\begin{aligned}
M & =\left[\begin{array}{cccc}
1 & 0 & 0 & 0 \\
0 & 91 & -76 \cos \left(x_{1}\right) & 0 \\
0 & -76 \cos \left(x_{1}\right) & 124.7 & 0 \\
0 & 0 & 0 & 1
\end{array}\right], \\
f_{1} & =x_{2}, \\
f_{2} & =744.8 \sin \left(x_{1}\right)+48 u \\
f_{3} & =200 u-76 x_{2}^{2} \sin \left(x_{1}\right) \\
f_{4} & =x_{3} \\
g & =x_{1}
\end{aligned}
$$

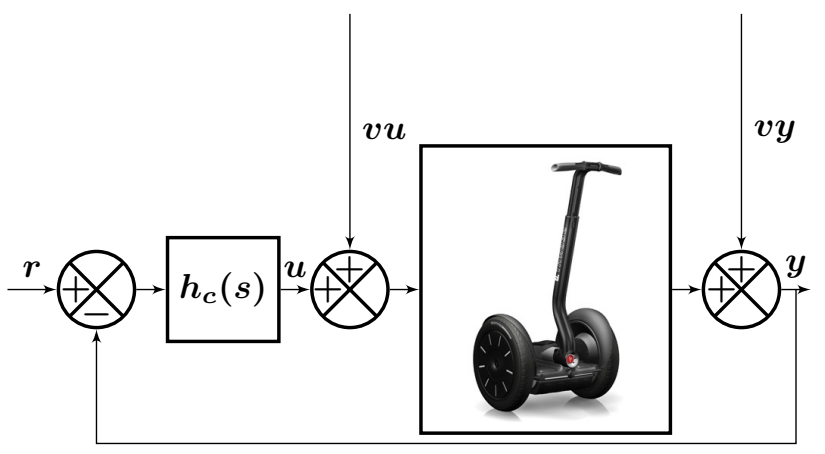

Figure 14: Example 3.8. Feedback system. Segway, and PID controller, $h_{c}(s)$. The input, $u$, is the motor drive voltage, $y$, is the tilt angle, $v u$, is input additive disturbance and, $v y$, is the output additive disturbance.

We will consider the input-output case defined as,

$$
\begin{aligned}
& u \in \mathbb{R}:=\{u: \text { motor signal }(V), \\
& y \in \mathbb{R}:=\{y: \text { tilt angle (rad), }
\end{aligned}
$$

Table 11: Example 3.8. Results from step 2 in Alg. 2.1, i.e. optimal DIPTD model approximation, where $c_{1}=0$ and $c_{2}=10$.

\begin{tabular}{|l|l|l|}
\hline \hline Method & $\hat{p}$ & $V(\hat{p})$ \\
\hline \hline Alg. 2.1 & $4.3347,0.0073,0.2163$ & $2.6528 \mathrm{E}-7$ \\
\hline
\end{tabular}

The simulation results are shown in Figure 15, where at Time $=0.1 \mathrm{~s}$, an output disturbance $v_{v y}=\frac{\pi}{18} \mathrm{rad}$ (10 deg) is introduced, and at Time $=1.0 \mathrm{~s}$, we give an input disturbance $v_{v u}=40 \mathrm{~V}$.

The indices given in Table 12 show similarities with previous Example 3.7, viz. Alg. 2.1 gives best result for $I A E_{v u}$, i.e. $\frac{I A E_{v u}^{A l g .2 .1}}{I A E_{v u}^{A l g .2 .1(S I M C)}}=1.5$ times better than Alg. 2.1 (SIMC) and Alg. 2.1 (SIMC) $\frac{I A E_{v y}^{A l g .2 .1}}{I A E_{v y}^{A l g .2 .1(S I M C)}}=1.1$ times better in terms of $I A E_{v y}$. 

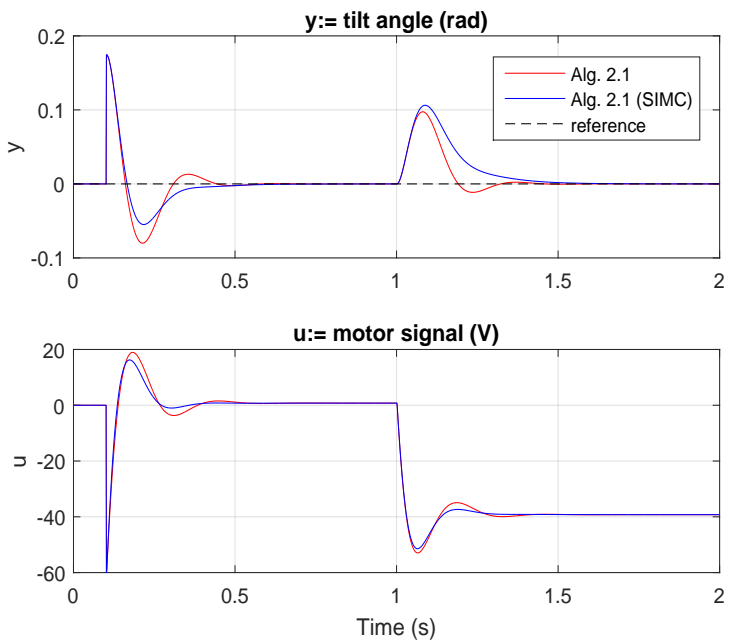

Figure 15: Example 3.8. Simulation of the Segway nonlinear model with PID control. Output and. input step disturbance at, Time $=0.1$ $\mathrm{s}$ and, Time $=1.0 \mathrm{~s}$, i.o. Input $u$ is limited.

Table 12: Example 3.8. Shows the indices $I A E_{v y}$, $I A E_{v u}, I A E$ (sum) and $T V$ for Alg. 2.1 where $\delta=1.6$, and Alg. 2.1 (SIMC) where $T_{c}=1.5 \tau$.

\begin{tabular}{|l|l|l|l|l|}
\hline \hline Method & $I A E_{v y}$ & $I A E_{v u}$ & $I A E$ & $T V$ \\
\hline \hline Alg. 2.1 & 0.0143 & 0.0112 & 0.0255 & 245.6765 \\
\hline Alg. 2.1 (SIMC) & 0.0128 & 0.0169 & 0.0297 & 223.5997 \\
\hline
\end{tabular}

\section{Example 3.9 (Nomoto Vessel)}

We will end this section by considering a Mariner vessel example. DIPTD model approximations for controller tuning for Mariner class vessel was demonstrated in Di Ruscio and Dalen (2017)) to be a successful approach.

In this example we will consider the nonlinear model proposed in Son and Nomoto (1982) which describes the motion of a high-speed container vessel of length $175 \mathrm{~m}$. This model has previously been implemented in the MSS GNC MATLAB Toolbox, Fossen and Perez (2004). We will consider the feedback system with added disturbances as illustrated in Figure 16.

Here, we are interested in an input-output case defined as,

$$
\begin{aligned}
& u \in \mathbb{R}:=\{u: \text { commanded rudder angle (rad), } \\
& y \in \mathbb{R}:=\{y: \text { yaw angle (rad). }
\end{aligned}
$$

The simulation results of the PID controller implementation are shown in Figure 1\%. The scenario is: at Time $=0 \mathrm{~s}$, the reference yaw angle is zero, at Time $=50 \mathrm{~s}$, we give the vessel an output disturbance step

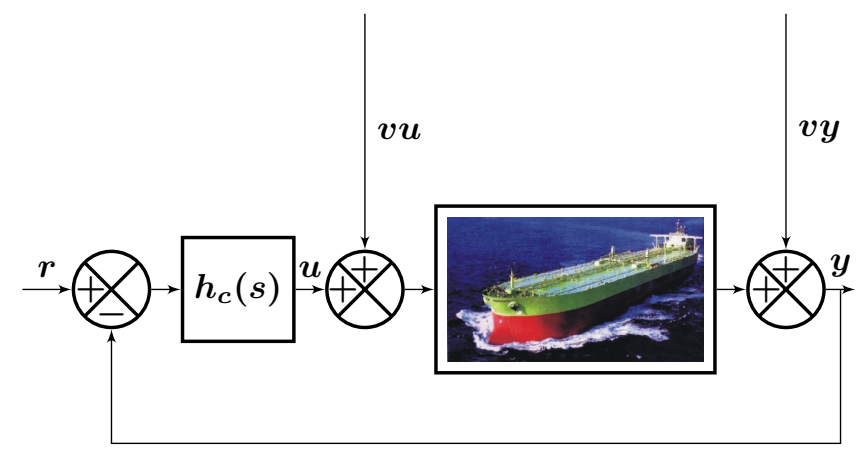

Figure 16: Example 3.9. Feedback system. Vessel model and controller $h_{c}(s)$. The input $u$ is the commanded rudder angle and the output $y$ is the perturbed yaw angle about zero. $v u$ is input additive disturbance and $v y$ is the output additive disturbance.

Table 13: Example 3.9. Results from step 2 in Alg. 2.1, i.e. optimal DIPTD model approximation, where $c_{1}=0$ and $c_{2}=1$.

\begin{tabular}{|l|l|l|}
\hline \hline Method & $\hat{p}$ & $V(\hat{p})$ \\
\hline \hline Alg. 2.1 & $0.0006,1.7867,7.3355$ & $1.6228 \mathrm{E}-7$ \\
\hline
\end{tabular}

$v_{v y}=\frac{\pi}{60} \mathrm{rad}(3 \mathrm{deg})$, at Time $=250 \mathrm{~s}$, we introduce an input step disturbance $v_{v u}=2 \frac{\pi}{9} \mathrm{rad}(40 \mathrm{deg})$.

We observe from Table 14 that Alg. 2.1 has an edge over Alg. 2.1 (SIMC) in terms of IAE $E_{v u}$, viz. $\frac{I A E_{v u}^{A l g .2 .1(S I M C)}}{I A E_{v u}^{A l g .2 .1}}=1.6$ times better. Note, the algorithms show approximately equal result for $I A E_{v y}$.

Table 14: Example 3.9. Shows the indices $I A E_{v y}$, $I A E_{v u}, I A E$ (sum) and $T V$ for Alg. 2.1 where $\delta=1.6$, and Alg. 2.1 (SIMC) where $T_{c}=1.5 \tau$.

\begin{tabular}{|l|l|l|l|l|}
\hline \hline Method & $I A E_{v y}$ & $I A E_{v u}$ & $I A E$ & $T V$ \\
\hline \hline Alg. 2.1 & 0.9930 & 0.3782 & 1.3712 & 2.9826 \\
\hline Alg. 2.1 (SIMC) & 0.9931 & 0.6140 & 1.6071 & 3.0588 \\
\hline \hline
\end{tabular}

\section{Discussion and Concluding Remarks}

The concluding remarks in this paper may be itemized as follows:

- The bounds, i.e. $A, b, l_{b}$ and $u_{b}$ in Eqs. (11)-(12) in Alg. 2.1 are partly ad-hoc based, it might be possible to make improvements on these. 

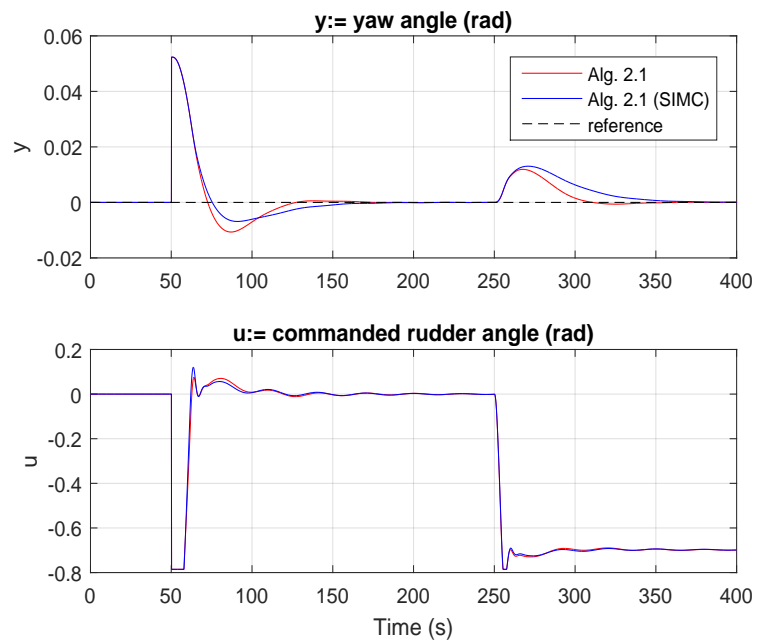

Figure 17: Example 3.9. Simulation of the nonlinear Nomoto Vessel model with PID control. Output and input step disturbance at, Time $=50 \mathrm{~s}$ and, Time $=250 \mathrm{~s}$, i.o. Input $u$ is limited.

- In Sec. 3.1 we have demonstrated successful implementations of the proposed model reduction technique Alg. 2.1 on a variety of linear models. We see from the stable part in Sec. 3.1 that Alg. 2.1 has an edge over the model based techniques SIMC and K-SIMC, viz., considering the optimality measures, $\Gamma_{m}$, in Table 6 (row 1:15, col. 4), the proposed Alg. 2.1 is at least $\frac{\Gamma_{S I M C}}{\Gamma_{A l g .2 .1}}=2.4$ times better than SIMC and K-SIMC.

- Note, that in Sec. 3.1 it is shown in Table 6 that SIMC is at least $\frac{\Gamma_{K-S I M C}}{\Gamma_{S I M C}}=1.2$ times better than K-SIMC, with the exception of Example 3.4.

- We see in Table 7 in the stable part in Sec. 3.1 that all the methods for prescribed robustness, $M_{s}=$ 1.59 , gives acceptable margins, i.e. $G M>1.7$ and $P M>30$ (Seborg et al. (1989)).

- Sec. 3.2 demonstrated successful implementations of Alg. 2.1 on unstable nonlinear models. In each example it is seen that Alg. 2.1 gives lower (sum) IAE indices than Alg. 2.1 (SIMC), where $\delta=1.6$ and $T_{c}=1.5 \tau$ were chosen, i.o.

Furthermore, Alg. 2.1 was shown to be at least $\frac{I A E_{v u}^{A l g .2 .1(S I M C)}}{I A E_{v u}^{A l g .2 .1}}=1.4$ times better in terms of input disturbance $I A E_{v u}$, however Alg. 2.1 (SIMC) was shown at least $\frac{I A E_{v y}^{A l g .2 .1}}{I A E_{v y}^{A l g .2 .1(S I M C)}}=1.2$ times better in terms of output disturbance $I A E_{v y}$. The exception is on the Nomoto vessel in Example 3.9 where both $I A E_{v y}$ were approximately equal.
- Note, that the derivative kicks are rather undesirable in practical applications. One possible solution is to use a low-pass filter on the derivative term, as in Example 3.6. If the process is stable, consider choosing a PI controller instead.

- It might be argued that, if the process model is known it might be the best solution to simply use the PO-PID controller.

\section{References}

Åström, K. and Hägglund, T. PID Controllers: Theory, Design, and Tuning. Instrument Society of America, 1995.

Åström, K. J., Panagopoulos, H., and Hägglund, T. Design of pi controllers based on non-convex optimization. Automatica, 1998. 34(5):585-601. doi:10.1016/S0005-1098(98)00011-9.

Balchen, J. A Performance Index for Feedback Control Systems Based on the Fourier Transform of the Control Deviation. Acta polytechnica Scandinavica: Mathematics and computing machinery series. Norges tekniske vitenskapsakademi, 1958.

Boubaker, O. The inverted pendulum: A fundamental benchmark in control theory and robotics. 2012. pages 1-6. doi:10.1109/ICEELI.2012.6360606.

Di Ruscio, D. Combined Deterministic and Stochastic System Identification and Realization: DSR - A Subspace Approach Based on Observations. Modeling, Identification and Control, 1996. 17(3):193-230. doi:10.4173/mic.1996.3.3.

Di Ruscio, D. Closed and Open Loop Subspace System Identification of the Kalman Filter. Modeling, Identification and Control, 2009. 30(2):71-86. doi:10.4173/mic.2009.2.3.

Di Ruscio, D. On Tuning PI Controllers for Integrating Plus Time Delay Systems. Modeling, Identification and Control, 2010. 31(4):145-164. doi:10.4173/mic.2010.4.3.

Di Ruscio, D. Pi controller tuning based on integrating plus time delay models: Performance optimal tuning. 2012. In Proceedings of the IASTED Control and Applications Conference. Crete Greece June 18-21.

Di Ruscio, D. and Dalen, C. Tuning PD and PID Controllers for Double Integrating Plus Time Delay Systems. Modeling, Identification and Control, 2017. 38(2):95-110. doi:10.4173/mic.2017.2.4. 
Fossen, T. I. and Perez, T. Marine Systems Simulator (MSS). 2004. URL http://www.marinecontrol. org.

Garpinger, O. and Hägglund, T. Modeling for optimal pid design. 2014. pages 6929-6934. Preprints of the 19th World Congress.

Jahanshahi, E., Oliveira, V. D., Grimholt, C., and Skogestad, S. A comparison between internal model control, optimal pidf and robust controllers for unstable flow in risers. IFAC Proceedings Volumes, 2014. 47(3):5752 - 5759. doi:10.3182/20140824-6ZA-1003.02381. 19th IFAC World Congress.

Jahanshahi, E. and Skogestad, S. Closed-loop model identification and pid/pi tuning for robust anti-slug control. IFAC Proceedings Volumes, 2013. 46(32):233 - 240. doi:10.3182/20131218-3-IN2045.00009. 10th IFAC International Symposium on Dynamics and Control of Process Systems.

Kotelnikov, V. A. On the transmission capacity of "ether" and wire in electrocommunications. Proc. 1st All-Union Conf. Technological Reconstruction of the Commun. Sector and Low-Current Eng, 1933. pages 1-19.

Lee, J., Cho, W., and Edgar, T. F. Simple analytic pid controller tuning rules revisited. Industrial \& Engineering Chemistry Research, 2014. 53(13):5038-5047. URL 10.1021/ie4009919, doi:10.1021/ie4009919.

LIngenieur, S. I. P. Comportement dynamique d'un vehicule auto-balance de type segway. Concours Centrale-Suplec, 2005. URL https://www. concours-centrale-supelec.fr/ CentraleSupelec/2005/PSI/sujets/SI.pdf. In french. Accessed 01.05.17.

Ljung, L. System Identification (2nd ed.): Theory for the User. Prentice Hall PTR, Upper Saddle River, NJ, USA, 1999.

MATLAB. Version 9.1.0.441655 (R2016b). The MathWorks Inc., Natick, Massachusetts, USA, 2016. Control System Toolbox, Version 9.3. Optimization Toolbox, Version 6.2.
Pareto, V. Il massimo di utilit dato dalla libera concorrenza. Giornale degli Economisti,luglio, 1894b. pages $48-66$.

Schmidt Z., J. P. . B., Brill. Choking can eliminate severe pipeline slugging. 1979. 312:230-238.

Seborg, D., Edgar, T., and Mellichamp, D. Process Dynamics and Control. Number v. 1 in Chemical Engineering Series. Wiley, 1989.

Seborg, D., Edgar, T., and Mellichamp, D. Process dynamics and control. Wiley series in chemical engineering. Wiley, 2004.

Shannon, C. E. Communication in the presence of noise. Proceedings of the IRE, 1949. 37(1):10-21. doi:10.1109/JRPROC.1949.232969.

Silva, G., Datta, A., and Bhattacharyya, S. PID Controllers for Time-Delay Systems. Control Engineering. Birkhäuser Boston, 2005.

Skogestad, S. Simple analytic rules for model reduction and PID controller tuning. Journal of Process Control, 2003. 13(13):291-309. doi:10.1016/S09591524(02)00062-8.

Skogestad, S., Havre, K., and Larsson, T. Control limitations for unstable plants. IFAC Proceedings Volumes, 2002. 35(1):485 - 490. doi:10.3182/200207216-ES-1901.00330. 15th IFAC World Congress.

Skogestad, S. and Postlethwaite, I. Multivariable feedback control: analysis and design. Wiley, 1996.

Son, K. H. and Nomoto, K. On the Coupled Motion of Steering and Rolling of a High Speed Container Ship. Naval Architect of Ocean Engineering, 1982. 20:73-83.

Yocum, B. Offshore Riser Slug Flow Avoidance: Mathematical Models for Design and Optimization. Society of Petroleum Engineers of AIME, 1973.

Ziegler, J. and Nichols, N. B. Optimum settings for automatic controllers. Trans. of the A.S.M.E., 1942. 64(64):759-768. 